susceptibility of patients to ventricular tachyarrhythmias. This technique could, therefore, help identify the patients with ischemic cardiomyopathy at the highest risk of sudden cardiac death, who might benefit most from ICD implantation.

Chow et al. enrolled 768 consecutive patients with ischemic cardiomyopathy and no history of ventricular arrhythmia and classified them as having negative (33\%) or non-negative (positive of indeterminate; 67\%) MTWA results at baseline. Of 376 patients without ICDs, 58 died during follow-up; 43 were MTWA non-negative, and 28 died from arrhythmic causes. In the ICD group, 41 of 392 patients died, of whom 35 were MTWA non-negative and 14 died from arrhythmic causes. Even following multivariable adjustment, patients with non-negative MTWA results had significantly greater risks of all-cause $(P=0.002)$ and arrhythmic $(P=0.049)$ mortality. MTWA did not predict nonarrhythmic mortality.

The authors conclude that MTWA strongly and independently predicts all-cause and arrhythmic mortality in patients with ischemic cardiomyopathy. They predict that, if added to the assessment of the already known risk factors for mortality in these patients, this technique could aid future risk stratification.

Original article Chow T et al. (2006) Prognostic utility of microvolt $\mathrm{T}$-wave alternans in risk stratification of patients with ischemic cardiomyopathy. J Am Coll Cardiol 47: 1820-1827

\section{Sirolimus-eluting stents exhibit incomplete neointimal coverage}

Sirolimus-eluting stents (SESs) inhibit neointimal hyperplasia, and are therefore associated with a lower risk of restenosis than bare-metal stents (BMSs). SES implantation might, however, increase the rate of late thrombosis, possibly because of impaired healing (incomplete neointimal coverage). Kotani et al. have compared neointimal coverage after SES implantation with that after BMS implantation.

Angioscopy was used to evaluate 37 consecutive stented coronary artery lesions in 25 patients (22 BMSs and 15 SESs). A new grading system was used to classify neointimal coverage, ranging from grade 0 (fully visible stent struts) to grade 3 (stent struts not visible by angioscopy).

Baseline demographics and lesion characteristics were similar in the two groups and the patients all received dual-antiplatelet therapy for a similar duration. Before and immediately after stent implantation, coronary angiographic findings did not differ between groups. At follow-up, 3-6 months after stent implantation, minimum lumen diameter was larger in the SES group than in the BMS group $(P=0.0255)$. Thrombi were identified in five SESs (33\%) and in three BMSs (14\%; $P=0.14)$. Neointimal coverage was significantly more complete with BMSs than with SESs: all 22 (100\%) BMSs had complete intimal coverage (grades 2 or 3 ), whereas only 2 of $15(13.3 \%)$ SESs had complete coverage $(P<0.0001)$. In an analysis including all 37 lesions, incomplete neointimal coverage was correlated with thrombus formation $(P=0.09)$.

The authors conclude that SES implantation appears to be associated with incomplete neointimal coverage and thrombus formation. They recommend that patients treated with SESs receive long-term dual antiplatelet therapy.

Original article Kotani J et al. (2006) Incomplete neointimal coverage of sirolimus-eluting stents: angioscopic findings.

J Am Coll Cardiol 47: 2108-2111

\section{Risk of cardiovascular disease very high in patients with type 1 diabetes}

Patients with type 1 diabetes are known to be at increased risk of cardiovascular disease (CVD), but the degree of risk is unknown. Strategies for disease prevention and evaluation of improvements in therapy are therefore difficult to develop. Soedamah-Muthu et al. have used information from the General Practice Research Database, which contains general practice records representative of the UK population, to estimate cardiovascular morbidity and mortality in a large cohort of patients with type 1 diabetes.

At study start, CVD prevalence was 3\% in patients with diabetes and $1 \%$ in the nondiabetic control group. Over the 7-year followup, type 1 diabetes was associated with a highly increased risk of major CVD: men with diabetes were four times more likely and women with diabetes were eight times more likely to experience a major event than controls. The risks of acute coronary events, coronary revascularizations, stroke, major coronary heart disease and fatal CVD were all greatly 